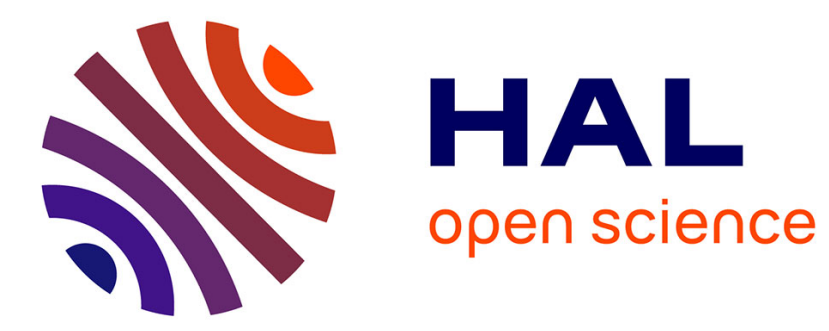

\title{
Un cas de changement phonologique par réanalyse morphonologique en éton.
}

Mark van de Velde

\section{To cite this version:}

Mark van de Velde. Un cas de changement phonologique par réanalyse morphonologique en éton.. Africana Linguistica, 2008, 14, pp.177-185. halshs-00627251

\section{HAL Id: halshs-00627251 https://shs.hal.science/halshs-00627251}

Submitted on 28 Sep 2011

HAL is a multi-disciplinary open access archive for the deposit and dissemination of scientific research documents, whether they are published or not. The documents may come from teaching and research institutions in France or abroad, or from public or private research centers.
L'archive ouverte pluridisciplinaire HAL, est destinée au dépôt et à la diffusion de documents scientifiques de niveau recherche, publiés ou non, émanant des établissements d'enseignement et de recherche français ou étrangers, des laboratoires publics ou privés. 
Un cas de changement phonologique par réanalyse morphonologique en éton Mark Van de Velde, Universiteit Antwerpen

\section{Abstract}

In the Cameroonian Bantu language Eton the stem of the possessive adjective 'my' ends in $/ \mathrm{o}$ / in the forms of class 1 and 3 (-amo) and in /a/ elsewhere (-ama). It will be shown that the original /a/ of the class $1 / 3$ forms changed to $/ 0 /$ due to a mechanism of analogical morphonological reanalysis.

\section{Résumé}

En éton, le thème de l'adjectif possessif de la première personne du singulier se termine en $/ \mathrm{a} /$ dans les formes de classe 1 et 3 (-amo) et en /a/ ailleurs (-ama). Cet article montre comment le /a/ originel des formes de classe 1 et 3 est devenu / $/$ / par un mécanisme de réanalyse morphonologique.

\section{Introduction}

Dans la langue bantoue camerounaise éton (A71), le thème des adjectifs possessifs de la première personne du singulier présente une irrégularité dans les accords de classe 1 et 3 . Ces formes ont une voyelle postérieure / $/$ / où les formes des autres classes d'accord ont /a/, voir le tableau 1 et les exemples (1-2). ${ }^{1}$

Tableau 1. Adjectifs possessifs de la première personne du singulier en éton

\begin{tabular}{|l|l|l|l|}
\hline I & w-àmò & II & b-ámà \\
\hline III & w-ámò & IV & mj-ámà \\
\hline V & d-ámà & VI & m-ámà \\
\hline VII & j-ámà & VIII & bj-ámà \\
\hline IX & j-àmà & X & j-ámà \\
\hline
\end{tabular}
/ǹ-nàm w-ámò/ 'mon pays' (classe 3)

${ }^{1}$ Cette analyse est basée sur mes donnés de terrain. Je tiens à remercier mes principaux informateurs Pie-Claude Ondobo et Désiré Essono, ainsi que Baudouin Janssens, Larry Hyman et deux lecteurs anonymes pour leurs suggestions. 
La présente contribution affirme que cette irrégularité dans la voyelle finale du thème est due à une réanalyse morphonologique de la séquence initiale $/ \mathrm{wa} /$. Malgré la présence d'une frontière morphologique entre la consonne préfixale / $\mathrm{w} /$ et la voyelle initiale du thème $/ a /$, la succession de ces deux phonèmes est réanalysée en tant que représentation d'un seul morphonème, nommément $|\mathrm{o}| \mathrm{La}$ voyelle $/ \mathrm{o} /$ dans la deuxième syllabe du thème est alors le résultat de l'application régulière des règles d'assimilation vocalique. Dans ce qui suit je montre d'abord que $|\mathrm{o}|$ est représentée par /wa/ dans certaines conditions, ce qui explique comment la réanalyse a pu se produire (section 2). La section 3 décrit ensuite la distribution phonotactique des morphonèmes vocaliques et les règles d'assimilation vocalique. Enfin, la section 4 donne des exemples de cas où la règle $\mathrm{d}$ 'assimilation vocalique interprète des séquences de phonèmes /wa/ comme étant la représentation d'une voyelle $|\rho|$, tandis que la forme sous-jacente de ces séquences doit être $|\mathrm{wa}|$.

Le modèle descriptif utilisé ici est constitué de trois niveaux d'analyse : un niveau morphonologique, un niveau phonologique et un niveau phonétique. Le passage du niveau morphonologique au niveau phonologique est réglé par des règles de représentation. La spécification du contexte dans les règles de représentation est strictement morphonologique. Les règles de représentation s'appliquent une seule fois, simultanément. Le contexte morphonologique ne change donc pas chaque fois qu'une règle est appliquée. Entre le niveau phonologique et phonétique il y a des règles de réalisation, dont la spécification du contexte est toujours strictement phonologique. Ces règles s'appliquent également une seule fois, simultanément.

L'éton peut être décrit avec 8 phonèmes vocaliques: /i, e, $\varepsilon, \partial, a, o, o, u /$ et la longueur vocalique (Van de Velde $2008: 15$ ). Dans les préfixes, /o/, /o/ et /e/ sont absents, tandis que $/ \varepsilon /$ et $/ \mathrm{a} / \mathrm{y}$ sont en distribution complémentaire $(/ \varepsilon /$ dans les préfixes de type $\mathrm{V}$ - et /a/ dans les préfixes de type $\mathrm{CV}-$ ). La distribution des voyelles dans le thème sera discutée dans la section 3 .

\section{Règles de représentation de $|0|$}

Un nombre de morphèmes est parfois réalisé avec une voyelle postérieure [0], parfois avec une succession semivoyelle-voyelle [wa]. Cette variation peut être libre, ${ }^{2}$ comme dans les exemples en (3) (le symbole $\sim$ sépare des variantes libres), mais elle peut aussi être conditionnée par le contexte morphonologique, comme dans les exemples en (4-6). La voyelle du radical |só| 'venir' est représentée par la voyelle $/ \mathrm{o} /$ à l'infinitif en (4a), et par/wa/ devant le suffixe |-gà| de la forme du passé d'hier en (4b). Il en va de même en (5-6), où $|\rho|$ est représentée par /o/ en

${ }^{2}$ Variation libre veut dire ici 'pas conditionnée par le contexte morphonologique ou phonologique'. Il peut y avoir un conditionnement dialectal, social ou idiolectal. 
position finale de mot $(5 \mathrm{a}, 6 \mathrm{a})$, et par /wa/ devant un suffixe dérivationnel (5b-c, $6 b)$. La représentation de la voyelle finale des formes en (3b), (4b), (5c) et (6b) sera expliquée dans la section 3.

(3) a. $\mid$ á d-kòm $\mid \rightarrow$ /á kòm á kwàm / $\rightarrow$ [á kòm á kwàm] 'faire, effectuer'

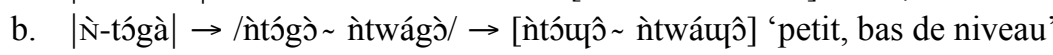

(4) a. $\mid$ á d-só $\mid \rightarrow$ lá `só/ $\rightarrow$ [á ^ só] 'venir' (forme de citation)

b. |bá-H-só-gà $\mid \rightarrow$ /báswág̀̀/ $\rightarrow$ [báswáûô] 'ils sont venus’

(5) a. $\mid$ á d-bò $\mid \rightarrow$ /á bò/ $\rightarrow$ [á bò] 'pourrir' (forme de citation)

b. $\mid$ ì-bò-lH $\mid \rightarrow$ /ìbwăl/ $\rightarrow$ [ìbwăl] 'pourriture'

c. |á d-bò-là $\mid \rightarrow$ /á bwàlò/ $\rightarrow$ [á bwàlò] 'faire pourrir' (forme de citation)

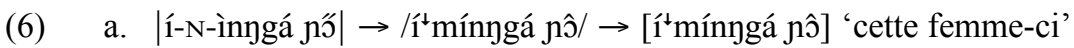

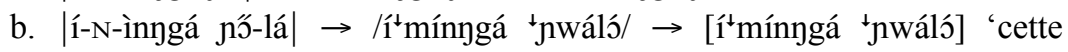
femme-là'

Il y a en principe deux analyses morphonologiques possibles pour les exemples en (3-6) : ou bien /wa/ représente un morphonème $|\rho|$, ou bien / $/ /$ est une représentation d'une succession de morphonèmes $\mid$ wa $\mid$. L'analyse retenue ici est la première, comme le montrent les notations morphonologiques dans les exemples. Le fait que /wa/ peut être la représentation d'une voyelle structurelle est démontré, entre autres, par la forme du préfixe nominal de classe 5 . Ce préfixe a deux allomorphes, nommément $|\grave{\varepsilon}-|$ qui apparaît devant les thèmes à initiale consonantique (7a) et $|\mathrm{d}-|$ pour les thèmes à initiale vocalique (7b-c). Dans les noms en (8), le choix des allomorphes du préfixe de classe 5 montre que la séquence /wa/ est parfois la représentation d'une voyelle structurelle (8a), parfois la représentation d'une séquence structurelle consonne-voyelle (8b). Un autre argument pour analyser /wa/ en tant que représentation de $|\rho|$ dans les exemples (3-6) est la règle d'assimilation vocalique discutée dans la section 3.
a. $\mid \grave{\varepsilon}$-tǐn $\mid \rightarrow$ /Ètǐy/ 'noeud'
b. $|\mathrm{d}-\mathrm{o} b| \rightarrow / \mathrm{dǒb} /$ 'ciel'
c. $\mid$ d-ùlà $\mid \rightarrow$ /dùlà/ 'promenade'
a. $\mid$ d-ǒd $\mid \rightarrow /$ dwăd/ $\rightarrow$ [dwădo'] 'furoncle'

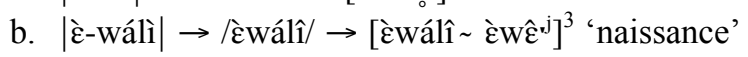

\footnotetext{
${ }^{3}$ La réalisation optionnelle [غेw $\left.\hat{\varepsilon}^{\mathrm{j}}\right]$ est due à la chute optionnelle de la consonne $/ 1 /$, suivie de l'assimilation vocalique.
} 
La représentation de $|\rho|$ est réglée comme suite. Le morphonème $|\rho|$ est obligatoirement représenté par $/ \mathrm{o} /$ devant une limite de mot (9). Ailleurs $|\rho|$ est représenté par $/ \mathrm{o} /$ ou $/ \mathrm{wa} /$ (selon les cas en variation libre, lexicalement conditionnée ou dialectalement conditionnée), avec une préférence pour / $/$ / devant une vélaire nasale (10), une certaine préférence pour /wa/ devant la consonne vélaire orale $/ \mathrm{g} /(11)$ et une très forte préférence pour /wa/ devant toute autre consonne (12). Pour comparaison, les exemples suivants montrent aussi les mots apparentés dans la langue bulu (A74), où $|0|$ est toujours représenté par / $/$.

\begin{tabular}{|c|c|c|}
\hline éton & bulu & traduction \\
\hline a. /bว̌/ & boo & 'cervelle' \\
\hline b. /î̀só/ & eso & 'ton père' \\
\hline c. /wว̀/ & wo & 'toi' \\
\hline d. /mbó/ & mvoy & 'graine' \\
\hline a. /દેđう̀̀/ & a-yэy & 'clan' \\
\hline b. /èbón غ̀bwán/ & a-boy & 'genou' \\
\hline c. /ìngòn/ & e-ngoy & 'oesophage' \\
\hline d. /ìnว̌y/ & e-non & 'lit' \\
\hline e. /ỳkwáy/ & n-koy & 'robinet (ét.), crête (bul.)' \\
\hline (11) a. /pwág/ & fok & 'un $(1)^{\prime 4}$ \\
\hline b. /कwág/ & jok & 'nager' \\
\hline a. /ngwàn/ & ngon & 'lune' \\
\hline b. /ỳkwăl/ & nkol & 'corde' \\
\hline c. /đ̧wáz/ & jos & 'juger' \\
\hline d. /îlwábí/ & e-lobe & 'marais' \\
\hline e. /îlwàlì/ & e-lole & 'canard' \\
\hline f. /itwàm/ & e-tom & 'rixe (ét.), palabre (bul.)' \\
\hline
\end{tabular}

\section{Assimilation vocalique}

L'éton a en commun avec beaucoup d'autres langues bantoues du nord-ouest que la première syllabe du thème prosodique y est proéminente. ${ }^{5}$ Un des domaines où cette proéminence se manifeste est la distribution phonotactique des morphonèmes (voir Van de Velde 2008: 17-23 pour une description plus approfondie de la proéminence syllabique). Nous avons déjà vu dans l'introduction que l'inventaire des phonèmes vocaliques est restreint dans les préfixes. L'occurrence des

\footnotetext{
${ }^{4}$ Les obstruantes sont dévoisées devant une pause en éton. Apparemment la notation des formes bulu donnée par Alexandre (1966) est phonétique.

${ }^{5}$ Le thème prosodique correspond plus ou moins au thème/radical morphologique. Il y a quelques exceptions, par exemple où le thème morphologique commence en une voyelle. Un préfixe consonantique fait alors partie du thème prosodique,

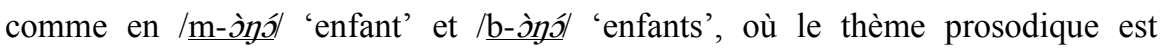
souligné et le thème morphologique est en italiques.
} 
phonèmes vocaliques dans le thème en dehors de la syllabe proéminente est soumise à des restrictions encore plus fortes. Le tableau 2 montre la distribution des phonèmes vocaliques dans les thèmes et radicaux dissyllabiques de structure (C) $\mathrm{VCV}^{6}$

Tableau 2. Distribution des phonèmes vocaliques dans les thèmes/radicaux

\begin{tabular}{|c|c|c|c|c|c|c|}
\hline $\mathrm{V}$ & $\mathrm{i}$ & $\mathrm{u}$ & $\mathrm{o}$ & $\varepsilon$ & 0 & $\mathrm{a}$ \\
\hline $\mathrm{i}$ & + & - & - & - & - & + \\
\hline $\mathrm{u}$ & + & - & - & - & - & + \\
\hline $\mathrm{o}$ & + & - & + & - & - & - \\
\hline$\varepsilon$ & + & - & - & - & - & + \\
\hline 0 & + & - & - & - & + & - \\
\hline $\mathrm{a}$ & + & - & - & - & - & + \\
\hline
\end{tabular}

Le tableau montre que seuls quatre phonèmes vocaliques peuvent apparaître en position $\mathrm{V}_{2}$ dans les thèmes dissyllabiques : $/ \mathrm{a} /, \mathrm{i} /, / \mathrm{o} /$ et $/ \mathrm{o} /$. En plus, l'apparition de $/ 0 /$ et $/ \mathrm{o} /$ en position $\mathrm{V}_{2}$ est soumise à deux conditions : $\mathrm{V}_{2}$ doit être le noyau d'une syllabe ouverte et la voyelle précédente doit être une voyelle du même timbre. Notez également que $\mathrm{V}_{2}$ ne peut pas être /a/ dans une syllabe ouverte si $\mathrm{V}_{1}$ est $/ \mathrm{o} / \mathrm{ou} / \mathrm{o} /$. Cette distribution des phonèmes vocaliques dans les thèmes peut être décrite avec la généralisation morphonologique que les seuls morphonèmes vocaliques qui existent dans le thème en dehors de la syllabe initiale sont $|\mathrm{i}|$ et $|\mathrm{a}|$, et une règle qui stipule que $|\mathrm{a}|$ est représenté par une voyelle harmonique dans une syllabe (de thème) finale, ouverte et non proéminente si la voyelle de la syllabe précédente est $|\mathrm{o}|$ ou $|\mathrm{o}| .^{7}$

(13) $|\mathrm{a}| \rightarrow / \mathrm{o} / /|\mathrm{o} \mathrm{C}(\mathrm{C}) \ldots \#|$

$$
|\mathrm{a}| \rightarrow / \mathrm{o} / /|\mathrm{o} C(\mathrm{C}) \ldots \#|
$$

La validité de cette règle d'assimilation vocalique peut être démontrée à l'aide des suffixes qui se terminent en $|a|$, tels que le suffixe statif $\mid$-bà| en (14), le suffixe

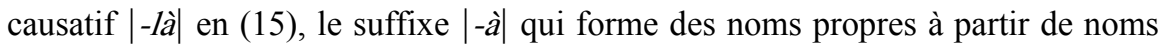
communs (16), et le suffixe agentif $\mid$-à| en (17)

(14) a. /nàl-bà/ 'mentir' (/ǹ-năl/ 'mensonge' (3))

b. /bún-bà/ 'être boudeur' (/màbún/ 'bouderie' (6))

c. /zín-bà/ 'être rancunier' (/zín/ 'haine' (9))

${ }^{6}$ Les phonèmes /e/ et /a/ n'apparaissent pas dans les thèmes dissyllabiques et ne figurent donc pas dans le tableau.

${ }^{7}$ Dans les thèmes trisyllabique chaque voyelle après $\mathrm{V}_{1}$ est $/ \mathrm{a} /$. 
d. /Ģòm-bò/ 'vieillir' (/̧̀nóm/ 'vieillard' (3))

e. /ndwàg-bذ̀/ 'être gourmand' (/ndwàg/ 'gourmandise' (9))

f. /tón-bô/ 'être lent' (/àtón/ 'lenteur' (3))

(15) a. /bá/ 'cuire (intr.)' ; /bélà/ 'cuire (tr.) ${ }^{8}$

b. /nùm/ 'puer'; / nùmlà/ 'sentir'

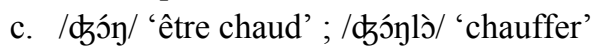

(16) a. /ùbàm/ 'épervier' ; /ùbàmà/ 'Epervier'

b. /Ènwăn/ 'oiseaux'; /

(17) a. /sùm/ 'labourer'; / ǹsùmà/ 'laboureur'

b. /sòm/ 'chasser'; ; 'ǹsòmò/ 'chasseur'

Les formes en (18-19) montrent l'application de la règle dans |-gà|, un suffixe flexionnel qui apparaît dans plusieurs formes verbales, dont le passé perfectif d'hier et le participe présent. Notez que l'exemple (19b) illustre également la règle de représentation de $|\rho|$ par /wa/.

(18) a. /á dù/ 'baptiser'; /bádûgà/ 'ils l'ont baptisé'

b. /á 'dí/ 'manger'; /bádígâ/ 'ils ont mangé'

c. /á bà/ 'découper'; /bábâgà/ 'ils l'ont découpé

d. /á ‘bó/ 'vomir'; /báçógô/ 'ils ont vomi'

(19) a. $\mid a ́$ d-ló $\mid \rightarrow$ /á ‘lós/ 'appeler' (forme de citation)

b. |bá-H-ló-gà $\mid \rightarrow$ /bálwágô/ 'ils l'ont appelé'

Enfin, les deux règles de représentation en question peuvent être observés dans l'adaptation de l'emprunt dollar en éton.

(20) $\mid$ dólà $\mid \rightarrow /$ dwál’̀ $/ \rightarrow$ [dwálô] 'pièce de cinq cents' < pidgin anglais dolla(r)

\section{Réanalyse morphonologique : $\mid$ wa $\mid$ est réanalysé comme $|\boldsymbol{0}|$}

Les règles morphonologiques décrites dans les sections 2 et 3 créent un gabarit prosodique qui fait qu'on s'attend à une voyelle $/ \mathrm{o} /$ plutôt que $/ \mathrm{a} /$ dans la deuxième syllabe d'un thème $\operatorname{CVC}(\mathrm{C}) \mathrm{V}$ si la première syllabe contient la séquence /wa/. L'existence d'un tel gabarit peut être déduit d'un nombre de formes où un $|\mathrm{a}|$ est représenté par /0/ tandis que la séquence /wa/ précédente ne peut pas être la

\footnotetext{
${ }^{8}$ Le phonème /a/ est réalisé comme une voyelle centralisée [e] dans une syllabe de thème non proéminente ouverte quand le noyau de la syllabe proéminente

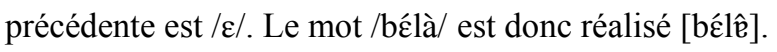


représentation d'un $|\rho|$ sous-jacent. En d'autres mots, la règle d'assimilation vocalique d'un $|\mathrm{a}|$ final montre que les séquences /wa/ qui sont la représentation de $\mid$ wa $\mid$ sont réanalysées en tant que représentations de $|0|$. Les radicaux verbaux en (21) illustrent cette réanalyse. La forme morphonologique retenue pour ces radicaux commence en $|w|$, plutôt que $|\rho|$, parce que aucun autre radical verbal commence en une voyelle en éton. Etant donné que la voyelle sous-jacente de la première syllabe de ces verbes est $|\mathrm{a}|$, la voyelle finale devait être /a/ selon les règles d'assimilation vocalique en l'absence d'une réanalyse morphonologique.

(21) a. |wágà $\rightarrow$ /wágう̀/ 'se laver' ; */wágà/

b. |wàdnà $\mid \rightarrow /$ wàdnう̀/ 'laver qn. avec des herbes médicinales' ; */wàdnà/

Le substantif de classe 5 èwágbó 'prétexte' est un autre exemple de réanalyse (22). $\mathrm{La}$ forme structurelle $\mathrm{du}$ thème doit commencer en un morphonème consonantique, puisque l'allomorphe $|\grave{\varepsilon}-|$ du préfixe nominal de classe 5 est sélectionné. Si le thème aurait commencé en $|0|$, le préfixe nominal de classe 5 devrait être |d-| (voir la discussion des exemples 7-8).

$$
\text { |غ̀-wágbá| } \rightarrow \text { /Èwágbó/ 'prétexte' }
$$

\section{Conclusion}

Trois éléments sont nécessaires pour expliquer pourquoi les adjectifs possessifs de la première personne du singulier se terminent en $/ \grave{\partial}$ / en classe 1 et 3 , tandis que les autres classes ont /à/: (i) la règle de représentation de $|\rho| \mathrm{par} / \mathrm{wa} /$ discutée dans la section 2 ; (ii) la règle de représentation de $|\mathrm{a}|$ par $/ \mathrm{0} /$ discutée dans la section 3 ; et (iii) le gabarit prosodique (C)waC(C)o qui résulte de l'application récurrente de ces deux règles, analysé comme une réanalyse morphonologique dans la section 4. Dans le cas des adjectifs possessifs, la réanalyse morphonologique est d'autant plus remarquable qu'il y a une frontière morphologique entre $|\mathrm{w}|$ et $|\mathrm{a}|$.

Dans ce qui précède, je n'ai pas proposé un ensemble de règles phonologiques qui réparent les perturbations dans l'application des règles générales causées par le phénomène de réanalyse morphonologique décrite ci-dessus. Selon le modèle descriptif que l'on préfère, il y a plusieures manières de représenter ces faits dans une description synchronique. Aucune de ces manières n'est en principe plus correcte que l'autre. Toutefois, on peut donner la préférence à une formalisation qui met en évidence le mécanisme de réinterprétation morphonologique entrainé par le gabarit prosodique / $\mathrm{wac} /$ très récurrent dans la langue, en évitant donc une solution dans laquelle une succession de règles donne "par hasard» le bon résultat. Dans le modèle morphonologique retenu pour la description de l'éton, on pourrait ainsi incorporer la règle de représentation schématique donné en (23), où la voyelle soulignée signale le nœud d'une syllabe proéminente. Tout ce qui est 
spécifié dans une règle schématique a préséance sur les autres règles de représentation. Les éléments sous-spécifiés dans une telle règle -les $|\mathrm{C}|$ en (23)subissent les règles morphonologiques générales.

(23)

$$
|\mathrm{w}(-) \mathrm{ac}(\mathrm{C}) \mathrm{a} \#| \rightarrow / \mathrm{waC}(\mathrm{C}) \mathrm{d} /
$$

\section{Bibliographie}

Alexandre, Pierre. 1966. Système verbal et prédicatif du bulu (Cameroun). Paris. Klinksieck.

Van de Velde, Mark L. O. 2008. A Grammar of Eton. Berlin. Mouton de Gruyter. 